\title{
Systematic Equation Formulation
}

\author{
Lindberg, Erik
}

Published in:

Proceedings of the 18th European Conference on Circuit Theory and Design

Link to article, DOI:

10.1109/ECCTD.2007.4529761

Publication date:

2007

Document Version

Publisher's PDF, also known as Version of record

Link back to DTU Orbit

Citation (APA):

Lindberg, E. (2007). Systematic Equation Formulation. In Proceedings of the 18th European Conference on Circuit Theory and Design (pp. 974-977). IEEE. https://doi.org/10.1109/ECCTD.2007.4529761

\section{General rights}

Copyright and moral rights for the publications made accessible in the public portal are retained by the authors and/or other copyright owners and it is a condition of accessing publications that users recognise and abide by the legal requirements associated with these rights.

- Users may download and print one copy of any publication from the public portal for the purpose of private study or research.

- You may not further distribute the material or use it for any profit-making activity or commercial gain

- You may freely distribute the URL identifying the publication in the public portal

If you believe that this document breaches copyright please contact us providing details, and we will remove access to the work immediately and investigate your claim. 


\title{
Systematic Equation Formulation
}

\author{
Erik Lindberg, IEEE Lifemember \\ Ørsted•DTU Department, 348 Technical University of Denmark, \\ DK2800 Kongens Lyngby, DENMARK \\ E-mail: el@oersted.dtu.dk, Telephone: +45 4525 3650, Fax: +45 45880117
}

\begin{abstract}
A tutorial giving a very simple introduction to the set-up of the equations used as a model for an electrical/electronic circuit. The aim is to find a method which is as simple and general as possible with respect to implementation in a computer program. The "Modified Nodal Approach", $M N A$, and the "Controlled Source Approach", $C S A$, for systematic equation formulation are investigated. It is suggested that the kernel of the PSpice program based on $M N A$ is reprogrammed.
\end{abstract}

\section{INTRODUCTION}

If you want to analyze an electrical/electronic circuit (network) the first step is to formulate equations which describe the relationships between the variables chosen. The second step is the solution of the equations in the time and frequency domains. The third step is the evaluation of the solution.

In the past a lot of methods have been proposed e.g. mesh-, nodal and cut-set-formulations of various kinds. All formulations are based on "natural" laws of balance and preservation of energy. The goal here is of course to find a method which is as simple and general as possible with respect to implementation in a computer program.

The mesh formulation is useful in calculations on simple circuits by hand. It is based on the Kirchhoff Voltage Law, $K V L$, which states that the sum of the voltage drops around any loop visiting a set of nodes is zero. The method is difficult to apply for non-planar circuits.

The nodal formulation is based on the Kirchhoff Current Law, $K C L$, which states that the sum of the currents leaving a closed subset of a circuit must be zero, e.g. the sum of the currents leaving an element (a branch, a two-terminal) is zero or the sum of the currents leaving a node is zero. A closed subset of a circuit is a "super node" or a "multi terminal". The variables chosen in the basic nodal formulation are the node voltages, i.e. the node potentials with respect to an arbitrary reference, "ground". This imply that "short circuits", "ideal voltage sources" and "ideal current controlled sources" can not be handled. Furthermore inductive elements will give rise to integro-differential equations or second order differential equations.

Since about 1960 a large number of computer programs have been developed for circuits and systems analysis and design. Many of these programs are based on the SPICE program from Berkeley [1]. The equation formulation scheme of many of these programs is the "Modified Nodal Approach", $M N A$ [2], based on the node potentials as primary variables. For circuits which contain voltage sources and other branches whose currents are controlling variables, the $M N A$ introduce those branch currents as additional variables.

Unfortunately, although it is more than 30 years ago that the $M N A$ was published it is difficult to find a textbook which describe this equation formulation scheme.

The PSpice program [3] has become the dominating industrial standard for circuit and systems analysis. One of the features of the program is "Analog Behavioral Modeling", $A B M$. PSpice-equivalent $A B M$ parts can be classified as either "E" (Voltage Controlled Voltage Source) or "G" (Voltage Controlled Current Source) device types. The "E" part type provides a voltage output, and the " $G$ " device type provides a current output.

Unfortunately, there are no equivalent "F" (Current Controlled Current Source) or "H" (Current Controlled Voltage Source) part types in the part library because PSpice "F" and " $\mathrm{H}$ " devices do not support the $A B M$ extensions. Also the $\mathrm{E}$ and the $\mathrm{G}$ device types can not be controlled directly by the time derivative of the controlling signal (variable), instead a voltage source/capacitor implementation is used. This indicate that PSpice do not implement the $M N A$ in a systematic and simple way.

In the following the "Modified Nodal Approach", $M N A$, and the "Controlled Source Approach", $C S A$, for systematic formulation of the equations will be investigated.

\section{THE "MOdified NODAl ApProACH"}

In the classic paper by Ho et.al. [2] introducing the "Modified Nodal Approach", $M N A$, the circuit equations are formulated as follows. First the nodal equations are formulated as

$$
\mathbf{Y} \underline{\mathbf{V}}=\underline{\mathbf{J}}
$$

where $\mathbf{Y}$ is the node admittance matrix, $\underline{\mathbf{V}}$ is the node potential vector (the common datum voltages) and $\mathbf{J}$ the current source vector. For the circuits which contain voltage sources and other branches whose currents are controlling variables, the $M N A$ proceeds by introducing those branch currents as additional variables and the corresponding branch constitutive relations as additional equations. These branch currents are available as additional output variables. For a given circuit, the matrix dimension is simply the sum of the number of nodes excluding the ground node plus the number of currents as outputs. The $M N A$ matrix can in general be expressed in the form:

$$
\left[\begin{array}{cc}
\mathbf{Y}_{R} & \mathbf{B} \\
\mathbf{C} & \mathbf{D}
\end{array}\right]\left[\begin{array}{l}
\underline{\mathbf{V}} \\
\underline{\mathbf{I}}
\end{array}\right]=\left[\begin{array}{l}
\underline{\mathbf{J}} \\
\underline{\mathbf{F}}
\end{array}\right]
$$


where $\mathbf{Y}_{R}$ is a reduced form of the nodal matrix excluding the contributions due to voltage sources, current controlling branches, etc. B contains partial derivatives of the Kirchhoff current equations with respect to the additional current variables and thus contains \pm 1 's for the elements whose branch relations are introduced. The branch constitutive relations, differentiated with respect to the unknown vector, are represented by the matrices $\mathbf{C}$ and $\mathbf{D}$. Tables with "stamps" for network branches as e.g. coils $\mathrm{L}$ or capacitors $\mathrm{C}$ are given. For each branch, depending on whether its current is an output or not, its contributions to the matrix can simply be read from the table and stamped into the matrix according to its node number or current number just labeled. For each of the branch types (element types) $G, C$ and $J$ there are two stamps depending on whether the branch current is an output variable or not. Capacitances and inductances are considered only in the time domain.

The general aspects for the derivation of stamps are as follows. The branch current is always introduced as an additional variable for an inductor and a voltage source, either independent or dependent, and is thus readily available as an output variable. For current sources J, resistors R, conductances $\mathrm{G}$, and capacitors $\mathrm{C}$, this is only done under the following conditions:

1. if other nonlinear circuit elements depend on its current and

2. if the branch current is requested as an output variable.

An example network with 5 branches is used for illustration of the approach. One of the branches is a nonlinear conductance which is specified as a function of its own current, $G_{3}=G_{3}\left(I_{3}\right)$ and not as function of its own voltage. This imply that a pathological current source controlled by its own current is modeled and the branch relation stamp becomes complicated. This network will be discussed in connection with the "Controlled Source Approach" below.

\section{The"Controlled SOURCE ApproAch"}

There are two kinds of electrical systems: (1) Power systems for transport of energy and (2) Electronic systems for transport of information. If you want to analyze these systems the first step is to set-up a model based on a choice of variables i.e. you have to make assumptions concerning the kind and number of variables which are adequate for the analysis you want to make. The basic physical variables are charge $q$ and flux $\varphi$ which are difficult to measure, instead current $(i=d q / d t)$ and voltage $(v=d \varphi / d t)$ are used as basic variables. The Maxwell equations are the basic equations which can be used for modeling an electrical system. Our first assumption is that the system is quasi-stationary i.e. the size of the system is small compared to the wavelength of the signals so that a lumped element model could be used.

Our second assumption is that the lumped elements of the system can be modeled as controlled sources. There are four kinds of controlled sources: VCCS (Voltage Controlled
TABLE I

ADMITTANCE BRANCHES ARE MODELED AS CURRENT SOURCES WHERE $t$ MEANS TIME, $f(-)$ MEANS "SOME FUNCTION OF", $I_{B}$ MEANS "THE CURRENT OF BRANCH B", AND $V_{B}$ MEANS "THE VOLTAGE OF BRANCH B".

\begin{tabular}{|c|c|}
\hline Admittance branches: & \\
\hline current sources & $I_{B}=f\left(V_{B}\right)$ \\
\hline Conductance: $\mathrm{G}$ & $I_{G}=f\left(V_{G}\right)$ \\
\hline Capacitance: $\mathrm{C}$ & $I_{C}=f\left(\frac{d V_{C}}{d t}\right)$ \\
\hline Controlled current sources: I & $V C C S(g m), C C C S(\beta)$ \\
\hline Independent current source: $\mathrm{J}$ & $J=f(t)$ \\
\hline
\end{tabular}

Current Source), VCVS (Voltage Controlled Voltage Source), $C C C S$ (Current Controlled Current Source) and CCVS (Current Controlled Voltage Source).

A controlled source may be defined as a four-terminal element with two input terminals " $n i+$ " and " $n i-$ " and two output terminals "no+" and "no-". A branch between the input terminals defines the controlling variable as voltage or current of the branch. The output terminals define the controlled source as a voltage or a current source. If the terminals are short circuited as follows: " $n i+$ " $=$ " $n o+$ " and " $n i-^{\prime \prime}=$ "no-" then we have a simple two-terminal element (a branch). A conclusion is that the model may be described by a list of branches (controlled sources), a "net-list". If the controlling branch of a four-terminal element is listed before the controlled branch in the net-list, then it is possible to setup the equations by only one scan of the net-list without having to look for a controlling branch when a controlled branch is found.

The branches of a lumped element model of an electrical system may be divided into two types: admittance branches and impedance branches [4]. An admittance branch is a branch for which the branch voltage is the primary variable (the input, the excitation) and the current is the secondary variable (the output, the response). An admittance branch is a $C C C S$ or a $V C C S$, e.g. a trans-capacitor or a capacitor is a current source controlled by the time derivative of the imposed voltage. An impedance branch is a branch for which the branch current is the primary variable and the voltage is the secondary variable. An impedance branch is a $V C V S$ or a $C C V S$, e.g. a trans-inductor or an inductor is a voltage source

TABLE II

IMPEDANCE BRANCHES ARE MODELED AS VOLTAGE SOURCES WHERE $t$ MEANS TIME, $f(-)$ MEANS "SOME FUNCTION OF", $I_{B}$ MEANS "THE CURRENT OF BRANCH B", AND $V_{B}$ MEANS "THE VOLTAGE OF BRANCH B".

\begin{tabular}{|c|c|}
\hline Impedance branches: & \\
\hline voltage sources & $V_{B}=f\left(I_{B}\right)$ \\
\hline Resistance: $\mathrm{R}$ & $V_{R}=f\left(I_{R}\right)$ \\
\hline Inductance: $\mathrm{L}$ & $V_{L}=f\left(\frac{d I_{L}}{d t}\right)$ \\
\hline Controlled voltage sources: V & $V C V S(\mu), C C V S(r m)$ \\
\hline Independent voltage source: $\mathrm{E}$ & $E=f(t)$ \\
\hline
\end{tabular}


controlled by the time derivative of the imposed current.

Note that a number of admittance branches in parallel may be considered as a single composite admittance branch and that a number of impedance branches in series may be considered as a single composite impedance branch. In order to make the equation system more compact, we will choose the node voltages (potentials with reference to an arbitrary ground node) instead of the branch voltages as primary variables together with the currents of the impedance branches. If the current of an admittance element is a controlling current, then this current will be taken as a primary variable also by means of introducing an extra node and an impedance branch (a zero valued resistor) in series with this admittance branch.

Table I and table II define the circuit elements. Conductors $\mathrm{G}$ and resistors $\mathrm{R}$ are two-terminal loss elements. Capacitors $\mathrm{C}$ and inductors $\mathrm{L}$ are two-terminal memory elements. The independent sources $\mathrm{J}$ and $\mathrm{E}$ are two-terminal input signal elements. The controlled sources are four-terminal active elements which define a coupling from one branch to another. As mentioned it is obvious that the two-terminal elements may be described as controlled sources which define branch self control e.g. a capacitor is a current source which is defined as a function of the time derivative of the voltage across the current source. Notice also that controlled sources with time derivative control (e.g. $V A=C C V S=\operatorname{frm}\left(\frac{d I R}{d t}\right)$ ) can easily be handled. By means of these branches we are able to deal with the four basic physical quantities: Current, Voltage, Flux and Charge.

If we apply Kirchhoff's Current Law to a general node $i$ in the circuit incident with all types of branches, we get the following equation

$$
\sum(\text { all currents })=0
$$

or

$$
\begin{gathered}
\sum(\text { currents in admittance branches }) \\
+\sum(\text { currents in impedance branches })=0
\end{gathered}
$$

or

$$
\begin{gathered}
\sum I_{G}+\sum I_{C}+\sum I_{I}+\sum I_{J} \\
+\sum I_{R}+\sum I_{L}+\sum I_{V}+\sum I_{E}=0
\end{gathered}
$$

If we consider the linear case and introduce the node voltages $\mathrm{v}$ we get

$$
\begin{gathered}
G(i, j) *\left(v_{i}-v_{j}\right)+C(i, j) * \frac{d\left(v_{i}-v_{j}\right)}{d t} \\
+g m(i, j) *\left(v_{k}-v_{l}\right)+\beta(i, j) * I_{Z}(k, l) \\
+I_{Z}(i, j)=-I_{J}(i, j)
\end{gathered}
$$

If we consider the admittance part of the complete system in matrix notation we get

$$
\mathbf{Y} \underline{\mathbf{V}}+[\mathbf{A Z}+\beta] \underline{\mathbf{I}}=-\underline{\mathbf{J}}
$$

where $\underline{\mathbf{V}}$ is the vector of node voltages, $\underline{\mathbf{I}}$ is the vector of impedance branch currents, $\mathbf{Y}$ is the node admittance matrix of the admittance branches, $[\mathbf{A Z}+\beta]$ is the sum of the incidence matrix $\mathbf{A Z}$ of the impedance branches and the contribution $\beta$ from the current controlled sources and $-\underline{\mathbf{J}}$ is the contribution from the independent current sources.

If we apply the element relations to a composite impedance branch, we get in the linear case for the branch voltage

$$
\begin{gathered}
\left(v_{m}-v_{n}\right)=R(m, n) * i(m, n)+L(m, n) * \frac{d i(m, n)}{d t} \\
+\operatorname{rm}(m, n) * i z+\mu(m, n) *\left(v_{i}-v_{j}\right)+\operatorname{VE}(m, n)
\end{gathered}
$$

If we consider the impedance part of the complete system in matrix notation we get

$$
\left[-\mathbf{A} \mathbf{Z}^{t}+\mu\right] \underline{\mathbf{V}}+\mathbf{Z} \underline{\mathbf{I}}=-\underline{\mathbf{E}}
$$

where $\underline{\mathbf{V}}$ is the vector of node voltages, $\underline{\mathbf{I}}$ is the vector of impedance branch currents, $Z$ is the impedance matrix of the impedance branches, $\left[-\mathbf{A} \mathbf{Z}^{t}+\mu\right]$ is the sum of the transpose negative incidence matrix $\mathbf{A Z}$ of the impedance branches and the contribution $\mu$ from the voltage controlled voltage sources and $-\underline{\mathbf{E}}$ is the contribution from the independent voltage sources.

Combining equations 4 and 5 gives

$$
\left[\begin{array}{cc}
\mathbf{Y} & {[\mathbf{A Z}+\beta]} \\
{\left[-\mathbf{A} \mathbf{Z}^{t}+\mu\right]} & \mathbf{Z}
\end{array}\right]\left[\begin{array}{l}
\underline{\mathbf{V}} \\
\underline{\mathbf{I}}
\end{array}\right]=\left[\begin{array}{l}
\underline{\mathbf{- J}} \\
\underline{\mathbf{- E}}
\end{array}\right]
$$

The above equation system make up the complete hybrid formulation of the circuit equations. It is easily seen that the system may be considered as a simple extension of the normal node equation formulation. In the process of formulation and solution of the equation system it is of course not necessary to sort according to equation 6 .

The kernel of the solution of a nonlinear system is the solution of a linear system. By means of Taylor evaluation it is possible to use the above-mentioned equation formulation for any nonlinear system which may be described by means of a set of first order differential equations and a set of algebraic equations.

It is obvious that the statement about the numerically dominating diagonal elements holds only for the passive part of a general circuit. Especially ideal elements as the short-circuit or the ideal controlled voltage source may give rise to zero valued diagonal elements. However, if we restrict ourselves to use diagonal elements only as candidates for pivoting, it is easily seen that these zeros will be turned into non-zeros during the Gaussian elimination process due to the structure of the equation system. In general the demand on numerical accuracy implies that pivot elements outside the diagonal must be chosen. Notice that it is possible to interchange rows and columns independently. By proper ordering of variables (columns) and equations (rows) it is possible to minimize the number of operations and the demand on computer storage during the solution process (sparse matrix techniques). 


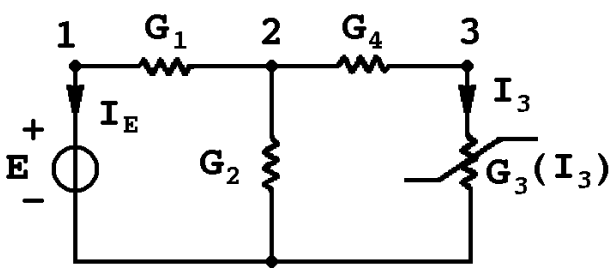

Fig. 1. Example network

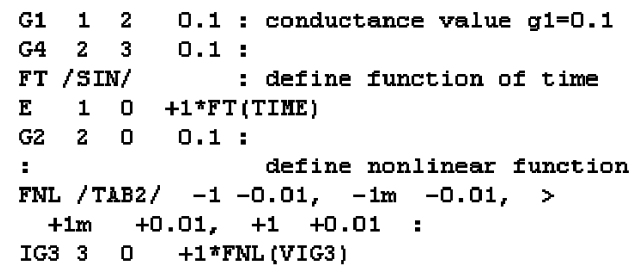

Fig. 2. Netlist for Example network

\section{AN EXAMPLE}

Figure 1 shows the example network used in the paper by Ho et.al. [2]. The circuit may be described by the netlist shown in Fig. 2. The conductance $G 3$ is modeled as a current source controlled by it own voltage instead of as function of the current as specified in the figure. A current controlled current source, $C C C S$, must be a four-/threeterminal element. It do not make sense to use the current both as response and as excitation (output = input). - The net-list is processed one element at a time. The extended node equations are created (grown) based on the following questions: Do we have new nodes (variables, equations) ? and What kind is this element (admittance, impedance) ?. The first branch $G 1$ give rise to two columns and two rows corresponding to node 1 and node 2. It is an admittance element, a conductance, so the value is added to the diagonal elements and subtracted from the off-diagonal elements defined by the nodes. The two rows are the equations defining Kirchhoffs current law $(K C L)$ for the two nodes. The second branch $G 4$ give rise to one new column (variable) and one new row $(K C L)$ corresponding to node 3 . The third branch is the independent voltage source $E$ (impedance) so the current $I_{E}$ give rise to a column and a row $(-V(1)=-E)$. The current leaves node 1 so we have +1 in row 1 and -1 in row 4 off-diagonal. In the diagonal we have a zero and in the right hand side column we have $-E$ as function of time. The branches $G 2$ and $I G 3$ are processed and the equation matrix and the right hand side of equation 6 becomes:

$$
\left[\begin{array}{cccc}
g 1 & -g 1 & 0 & +1 \\
-g 1 & g 1+g 4+g 2 & -g 4 & 0 \\
0 & -g 4 & +g 4+g 3(F N L) & 0 \\
-1 & 0 & 0 & 0
\end{array}\right]
$$

$$
\left[\begin{array}{c}
0 \\
0 \\
-J(F N L) \\
-E
\end{array}\right]
$$

where $g 3(F N L)$ is the linear instant dynamic conductance and $-J(F N L)$ is the equivalent linear instant current source in parallel with the conductance. Linearizing the nonlinearities by means of Taylor series evaluation means e.g. that a function $y=f(x)$ is replaced with $f(x)=f(0)+x * f d o t(0)$ where $f d o t=d y / d x$. In this case $y=I G 3=+1 * F N L(V I G 3)=f(x), g 3(F N L)=f d o t(0)$ and $-J(F N L)=f(0)$.

The equation formulation scheme mentioned above was implemented in the NAP2 program by the late Thomas RübnerPetersen in 1972 [5], [6], [7].

\section{CONCLUSION}

The modified nodal approach $M N A$ is compared with a simple strategy for setting up the equations for an electrical or electronic circuit, the "Controlled Source Approach", CSA. The strategy is based on the concept of admittance and impedance elements. All elements are interpreted as controlled sources. The equations may be sorted into two parts. The first part is based on Kirchhoffs current law for all the nodes of the circuit. This part contains all voltage controlled current sources and all current controlled current sources. The second part is based on Kirchhoffs voltage law for the impedance elements. This part contains all voltage controlled voltage sources and all current controlled voltage sources. MNA stamps are not necessary. SPICE-programs based on $M N A$ should be reprogrammed.

\section{REFERENCES}

[1] L.W. Nagel, "SPICE2: A Computer Program to Simulate Semiconductor Circuits", Memorandum No. ERL-M520, University of California, Berkeley, 1975.

http: //bwrc.eecs.berkeley.edu/Classes/IcBook/ SPICE/

[2] Ho, C.W., Ruehli, A.E., Brennan, P.A. “ The Modified Nodal Approach to Circuit Analysis ", IEEE Transactions on Circuits and Systems, Vol. CAS-22, June 1975, pp. 504-509.

[3] http://www. cadence.com/products/orcad/ pspice a d/index.aspx

[4] Erik Lindberg, "On the Formulation and Solution of Network Equations", Proceedings of the Fourth International Symposium on Network Theory, Ljubljana, Yugoslavia, September 4-7, 1979, pp. 427-432, YU ISSN 0351-1669.

[5] Thomas Rübner-Petersen, "The NAP2 integration algorithm - An Efficient Algorithm Using Backward Time-Scaled Differences For Solving Stiff Differential-Algebraic Systems", Institute of Circuit Theory and Telecommunication, 343 Technical University of Denmark, DK-2800 Lyngby, Denmark, September 1973.

[6] Thomas Rübner-Petersen, "NAP2 - Mathematical background. A preliminary report.", Institute of Circuit Theory and Telecommunication, 343 Technical University of Denmark, DK-2800 Lyngby, Denmark, 1974.02.22.

[7] Erik Lindberg and Thomas Rübner-Petersen, "The Theory behind NAP2", Institute of Circuit Theory and Telecommunication, 343 Technical University of Denmark, DK-2800 Lyngby, Denmark, Report IT-32 (2. edition), October 1981, ISSN 0105-8541. 